\title{
Glucocorticoid receptors: finding the middle ground
}

\author{
Sofie J. Desmet and Karolien De Bosscher
}

Receptor Research Laboratories, Nuclear Receptor Lab, Medical Biotechnology Center, VIB, Ghent, Belgium. Department of Biochemistry, Ghent University, Ghent, Belgium.

\begin{abstract}
Clucocorticoids (GCs; referred to clinically as corticosteroids) are steroid hormones with potent anti-inflammatory and immune modulatory profiles. Depending on the context, these hormones can also mediate pro-inflammatory activities, thereby serving as primers of the immune system. Their target receptor, the GC receptor (CR), is a multi-tasking transcription factor, changing its role and function depending on cellular and organismal needs. To get a clearer idea of how to improve the safety profile of GCs, recent studies have investigated the complex mechanisms underlying GR functions. One of the key findings includes both pro- and anti-inflammatory roles of GR, and a future challenge will be to understand how such paradoxical findings can be reconciled and how GR ultimately shifts the balance to a net anti-inflammatory profile. As such, there is consensus that CR deserves a second life as a drug target, with either refined classic GCs or a novel generation of nonsteroidal GR-targeting molecules, to meet the increasing clinical needs of today to treat inflammation and cancer.
\end{abstract}

\section{Introduction}

Glucocorticoids (GCs) are steroid hormones that are derived from cholesterol and secreted by the zona fasciculata of the adrenal glands. GC production occurs in a circadian- and stress-associated manner and is regulated by the hypothalamic-pituitary-adrenal (HPA) axis (1-3). Various physiologic processes, including glucose metabolism and metabolic functions in fat, muscle, and bone, are under GC control. Both natural and exogenous GCs function through activation of the GC receptor (GR), a transcription factor (TF) encoded by the NR3C1 gene. Alternative splicing and translation start sites (TSSs) give rise to several GR transcripts (4-6), with the full-length $\mathrm{GR} \alpha$ as the predominant active isoform.

GCs diffuse freely through the cellular membrane and bind to the cytoplasmic GR $\alpha$ complex. Ligand binding induces a conformational change in the receptor, exposing nuclear localization signals and (ex)changing interaction partners, after which the receptor translocates to the nucleus. HSP70/90-based chaperone machinery functions with cochaperones to guarantee proper folding, maturation, nuclear accumulation, and DNA binding of the receptor $(7,8)$. Further, chaperone and cochaperone composition of this complex alters GR sensitivity. For example, FK506 binding protein-5 (FKBP5) association with the receptor complex decreases affinity for cortisol and results in less efficient nuclear translocation $(9,10)$. Because of their role in GR activation, chaperones are now being investigated as potential drug targets in the pathophysiology of stress-related psychiatric disorders (9), but they may also be relevant in other disease settings where GR activities are important. Once in the nucleus, GR target genes (e.g., FKBP5) are activated via receptor homodimer binding onto cognate DNA sequences, termed GC response elements (GREs). This mechanism is referred to as transactivation. Investigating GR occupancy on GRE elements in A549 human lung cells showed that $63 \%$ of GREs are more than $10 \mathrm{~kb}$ from the TSS. Furthermore, both the

Conflict of interest: The authors have declared that no conflict of interest exists. Reference information: / Clin Invest. 2017;127(4):1136-1145.

https://doi.org/10.1172/JCl88886. core GR binding sequences as well as the GRE architecture harbor gene-specific regulatory information (11). Besides acting as a genuine TF, activated GR can also influence target genes in the nucleus via other mechanisms (ref. 12 and discussed below).

GCs are primarily used in the clinic for their potent antiinflammatory actions $(1,2,13)$. Indications range from short-term treatments for conditions such as skin rashes (14), seasonal allergic rhinitis (15) and relapses of multiple sclerosis (16) to long-term treatments for diseases such as severe asthma (17) and rheumatoid arthritis (18). GCs still suffer from a bad reputation in the clinic: patients fear the long-term consequences of GC-based therapy, not in the least because of the appearance-changing psychological impact caused by water retention, resulting in a typical moon face and imbalanced fat build-up (Figure 1 and ref. 19). Recently, the multidisciplinary European League Against Rheumatism (www. EULAR.org) critically reviewed the evidence on the four most worrisome adverse effects of GC therapy from the clinician's perspective, which are osteoporosis, hyperglycemia/diabetes mellitus, cardiovascular diseases, and infections. The risk of harm is especially elevated for patients taking long-term dosages equivalent to over $10 \mathrm{mg}$ prednisone per day; at dosages of 5 to $10 \mathrm{mg} / \mathrm{day}$, patient-specific characteristics determine the risk of harm (18).

In the current Review we focus on transcriptional mechanisms that explain how the intracellular mediator of GC actions, the GR, modulates gene expression to control inflammation. Furthermore, we emphasize the need for research into strategies to improve the safety profile of GRs.

\section{Anti-inflammatory effects via gene suppression} GCs exert their anti-inflammatory actions at multiple levels. At the cellular level, they suppress cytotoxic T lymphocytes (20) and impair DC maturation (21). Effects on immune and nonimmune cells involved in inflammation obviously differ. Historically, the bulk of GC anti-inflammatory effects were linked to GR-mediated gene suppression because the activated GR $\alpha$ typically interferes with the activities of various pro-inflammatory TFs, including $\mathrm{NF}-\kappa \mathrm{B}$, activator protein 1 (AP-1), and interferon regulatory factors 


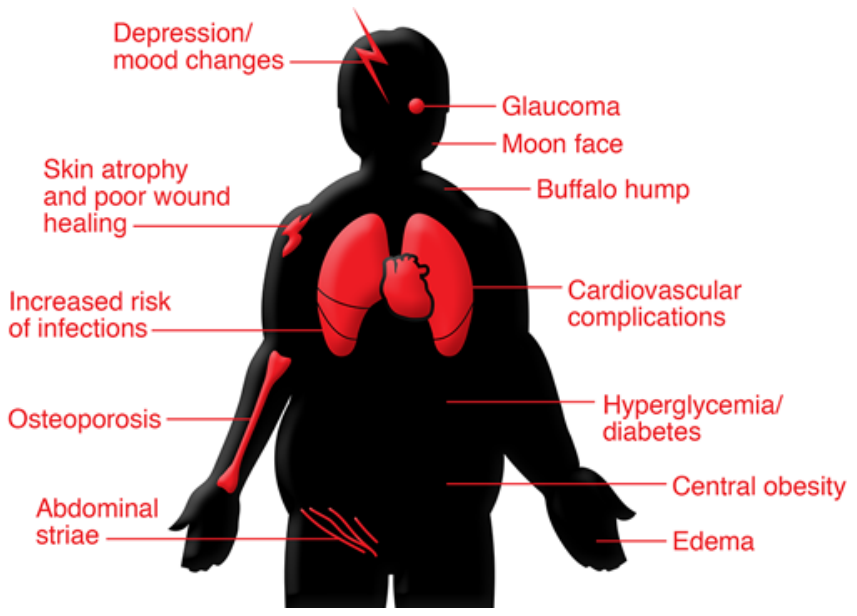

Figure 1. Graphic presentation of GC-associated side effects. GCs can lead to a number of burdening side effects, depicted here, typically when used at higher doses and for a longer period of time, as is done with chronic inflammatory diseases.

(IRFs) (22-24). The process by which one TF influences the activity of another DNA-bound TF without contacting DNA itself has been termed transrepression. GR-mediated transrepression can be achieved via differing mechanisms that depend on both cellular identity and promoter context $(12,25)$. For example, transrepression can involve a sequestration or squelching mechanism whereby the GR-targeted, DNA-bound TF detaches from the DNA, or a tethering mechanism whereby the GR remains associated with the DNA-bound TF (12). Over the years, subtle variants of the transrepression mechanism have been described. These include the following nonexclusive (and sometimes debated) mechanisms by which GR mediates repression of NF- $\kappa \mathrm{B}$ transcriptional activity: (a) GR-mediated tethering $(26,27)$ or squelching $(28)$ of the NF- $\mathrm{KB}$ p65 subunit, (b) recruitment of histone deacetylase 2 by deacetylated GR to NF- $\kappa \mathrm{B}$-dependent promoters (a specific consequence of tethering) (29), (c) disruption of p65/IRF complexes by GR (30) at RNA polymerase II (Pol II) initiation-controlled inflammatory genes in primary macrophages, and (d) GR-mediated blockade of positive transcription elongation factor- $\mathrm{b}$ ( $\mathrm{p}-\mathrm{TEFb}$ ) recruitment to Pol II, which impairs transcriptional elongation. This last mechanism exemplifies a selective repression by targeting a promoterspecific coregulator, in this case for the IL8 promoter. In contrast, NF- $\kappa \mathrm{B}-$ dependent induction of $\mathrm{I} \kappa \mathrm{B} \alpha$ gene expression is not dependent on p-TEFb and is therefore not suppressed by GR (31, 32). Interestingly, GR does not affect Pol II recruitment or transcription initiation at elongation-controlled genes but promotes accumulation of a pause-inducing negative elongation factor in a GR interaction protein 1 (GRIP1) cofactor-dependent manner (33). Rogatsky and colleagues recently demonstrated the importance of GRIP1 in homeostatic macrophage activation and function (34). GRIP1 was found to be involved in two distinct mechanisms in different macrophage populations: (a) GRIP1 cooperated with GR to assist in the transcriptional inhibition of inflammatory mediators by monocyte-derived macrophages and (b) GRIP1 cooperated with Krüppel-like factor-4 (KLF4) to effectively install a homeostatic transcription program in tissue-resident macrophages (34).
Alternative mechanisms that explain transcriptional repression include indirect mechanisms wherein GR competes with other TFs such as NF- $\mathrm{B}$, IRF3, or AP-1 for essential (co-)activators such as CREB binding protein, nuclear receptor coactivator 1, GRIP1, or p53 (35-41). Additionally, pioneering work by Yamamoto and colleagues demonstrated that GR can also bind to DNA recognition sequence half sites, i.e., only part of the classic palindromic GR-binding sequence motif, as a monomer (42). A recent study by Steger and colleagues showed that GR monomers bind DNA, thereby facilitating transient contacts with nearby TFs in a process known as half-site-facilitated tethering (43). An alternative role for the partner proteins with which GR can tether was proposed by Hager and colleagues, who identified AP- 1 as a facilitator of productive GR/chromatin interactions, following the observation that GR binding takes place at particular chromatin regions that are accessible even prior to hormone treatment (44). These studies demonstrate that GR is a versatile and mechanistically creative transcriptional repressor of the activity of other TFs.

\section{Anti-inflammatory effects via gene activation}

It has long been known that GR can activate genes encoding proteins that oppose different aspects of inflammatory signaling (45), e.g., lipocortin I (encoded by the ANXA1), the inhibitor of NF- $\mathrm{BB}$ (I $\mathrm{B} \mathrm{B} \alpha$, encoded by NFKBIA), and IL-10. A common feature of the GR-supported gene activation mechanism is that the effects are all indirect and involve increased production of specific antiinflammatory mediators. The contribution of this mechanism to the resolution of inflammation has historically been considered to be minimal; however, the primacy of GR-mediated transrepression of genes as a predominantly anti-inflammatory mechanism has been called into question by recent studies. GR-mediated transactivation of anti-inflammatory genes has been shown to be essential in curbing inflammatory diseases. Indeed, by inhibiting de novo protein synthesis and thereby curtailing anti-inflammatory protein production, the ability of GC to effectively resolve inflammation is attenuated (46-49). Furthermore, a number of candidate GR target proteins with anti-inflammatory function carry classic GRE elements in their promoters. For example, GCs induce the anti-inflammatory protein MAPK phosphatase 1 (MKP1, also referred to as DUSP1) (Figure 2), which dephosphorylates the MAPK p38 to inhibit pro-inflammatory gene expression (50-56). In vivo evidence supporting a role for GR dimerization in mediating the anti-inflammatory effects of GCs was provided by studies of GR ${ }^{\mathrm{dim} / \mathrm{dim}}$ mice (57), which express a GR that is compromised in its ability to form dimers. These mice are extremely sensitive to TNF-induced death due to their inability to induce Mkp1. In a TNF-induced acute inflammation model, MKP1 dephosphorylates the MAPK JNK2, thereby inhibiting intestinal epithelial cell apoptosis (58). These studies indicate that GCs act at different levels in the inflammation pathway to suppress ongoing inflammation, including via transrepression of pro-inflammatory TFs (as described above) and by targeting distal regulators such as MAPK kinases via upregulation of MKP1 (see above) or upregulation of $\mathrm{I} \kappa \mathrm{B} \alpha$, the endogenous inhibitor of NF- $\kappa \mathrm{B}$ (Figure 2 and refs. 59, 60). Moreover, at the posttranscriptional level, GCs destabilize pro-inflammatory cytokine mRNAs by inducing the zinc finger protein 36 (ZFP36, also known as tristetraprolin) (Figure 2 and ref. 


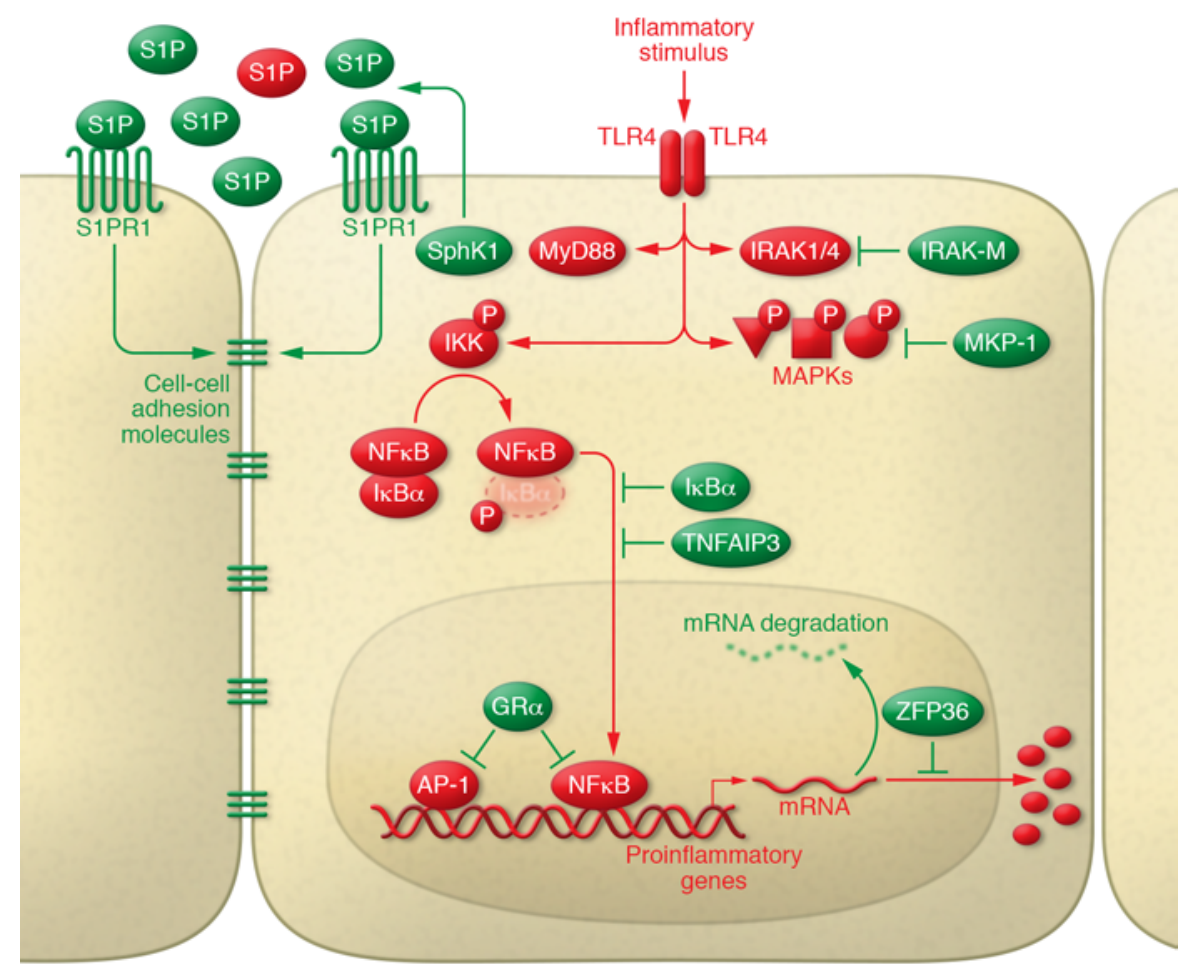

\begin{abstract}
Figure 2. GCs act at multiple levels in the inflammatory pathway. An inflamed cell is depicted, along with inflammatory mediators (red), which show a signal transduction pathway leading to the activation of NF- $\mathrm{KB}$ and $\mathrm{AP}-1$, which subsequently drive proinflammatory gene expression. GC-mediated and GR-triggered responses are depicted (green), including feedback and feedforward mediators, as described in the text. This includes upregulation and activities of various proteins, e.g., MKP-1, ZFP36, IRAK-M, and SphK1. IKK, IKB $\alpha$ kinase; IRAK, IL-1R-associated kinase-4; S1P, sphingosine-1-phosphate.
\end{abstract}

61). These may be cell-specific mechanisms, since an exclusively nuclear transcriptional mechanism that is independent of de novo protein synthesis was identified in other cell types (62).

In severe asthma and other inflammatory airway diseases that are commonly treated with GCs, TNF- $\alpha$ mRNA and protein expression is elevated $(63,64)$. To understand the impact of GCs on negative feedback control of TNF, Gerber and colleagues investigated how the expression of anti-inflammatory TNF targets such as TNF $\alpha$-induced protein 3 (TNFAIP3, encoding A20) and NFKBIA (encoding $\operatorname{I} \kappa \mathrm{B} \alpha$ ) is selectively spared or augmented by dexamethasone (DEX) treatment in airway epithelial cells. Their data fit a model in which the expression of anti-inflammatory targets of TNF is maintained during treatment with GCs through a context-dependent cooperation between GR and NF- $\mathrm{BB}(64)$. Along the same lines, Miyata and colleagues identified central regulators of innate inflammation, including myeloid differentiation factor 88 , the central adaptor molecule for TLRs and IL-1R family members, and IL-1R-associated kinase-1 (IRAK-1) and IRAK-4, as GC targets (65). The inactive kinase IRAK-M is induced upon TLR stimulation and negatively regulates TLR signaling (66). Notably, activated GR $\alpha$ also induces the expression of IRAK-M (Figure 2) in both airway epithelial cells and macrophages (65), thereby counteracting inflammatory TLR signaling. The GR monomer-inducing compound A $(67,68)$ could not enhance IRAK-M expression, suggesting a GR dimerization-dependent mechanism (65). These findings collectively show that, in addition to cytokine repression, GR-mediated induction of immune regulators can assist in halting pro-inflammatory signaling pathways at various levels of the signal transduction cascade.

Besides directly targeting pro-inflammatory pathways, GCs can also induce anti-inflammatory, protective mechanisms at the cellular level. For example, GCs promote the restoration of endo- thelial barrier integrity in the lung by inducing the expression of sphingosine kinase 1 (SPHK1) in both macrophages and endothelial cells, thereby inhibiting leukocyte infiltration. SPHK1 expression has proven to be crucial for GR-mediated anti-inflammatory actions in the lung, as downregulation of pro-inflammatory cytokines alone was not enough to resolve inflammation (69). Other anti-inflammatory proteins induced by GCs include the GCinduced leucine zipper (GILZ) (70, 71), the ectonucleotide pyrophosphatase/phosphodiesterase NPP1 $(72,73)$, annexin 1 (74), and the secretory leukocyte proteinase inhibitor (75). In trying to identify targets through which GCs exert therapeutic effects in asthma, Kadiyala and colleagues found that GR recruits p 65 to dimeric GR binding sites across the genome to augment gene expression. GR targets regulated by this mechanism include key anti-inflammatory and injury response genes such as SERPINA1 and FOXP4, the latter being an inhibitor of mucus production. Thus, cooperative anti-inflammatory gene regulation by GR and $\mathrm{p} 65$ contributes to GC efficacy, diminishing the role of GR/p65-tethering mediated gene repression as a means to resolve inflammation (76).

\section{Feedback and feedforward loops in GC signaling}

Pro-inflammatory pathways enhance many of the aforementioned GC-induced anti-inflammatory genes, thereby initiating feedback or feedforward loops. A well-known feedback loop triggered by inflammation is the augmented synthesis and secretion of GCs themselves via direct activation of the HPA axis by numerous proinflammatory cytokines (24). Additionally, DEX synergizes with nontypical Haemophilus influenzae to induce the binding of both GR and p65 at the promoter of Irakm, with both factors working cooperatively to attenuate bacteria-induced inflammation (65). Gene regulatory interactions were also identified by Vettorazzi and colleagues for several other pro-inflammatory stimuli (69). In combination 
A

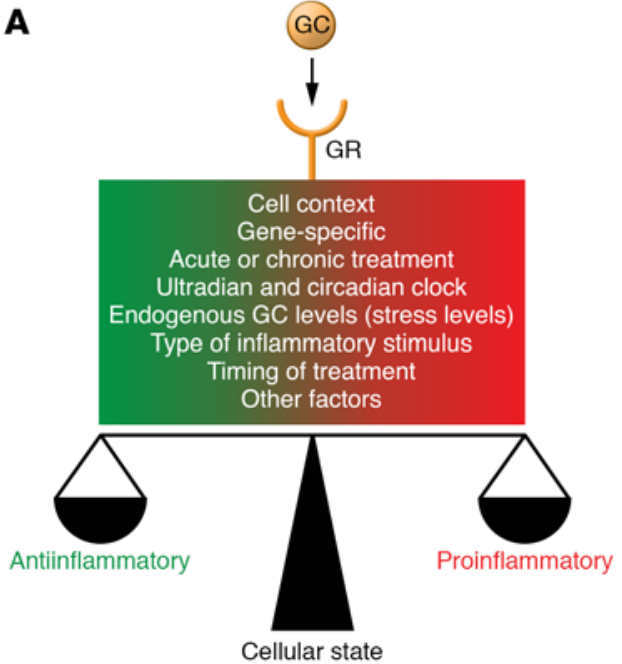

B
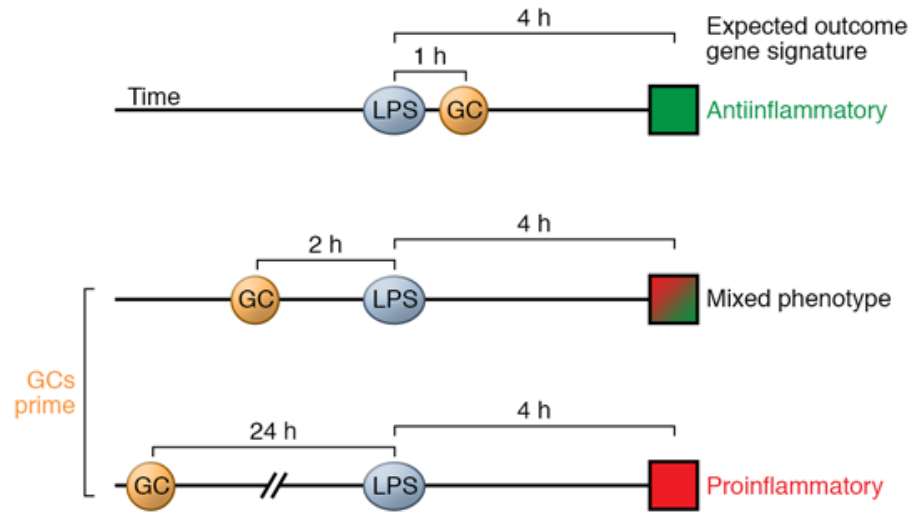

Figure 3. Influencing the effects of GCs. (A) Factors that may contribute to the ability of GCs to shift the balance toward a net pro-inflammatory or antiinflammatory cellular state. An incoherent control system that includes a proinflammatory role for GCs is essential to prevent excessive inflammation and to effectively return to homeostasis. This results in a continuous competition between feedback and feedforward control. (B) The timing of treatment may contribute to the ability of GCs to shift the balance toward a net proinflammatory or antiinflammatory cellular state. This insight is important for critically (re-)evaluating in vitro and/or in vivo studies in which GCs are combined with an inflammatory trigger, here exemplified by LPS, in a laboratory-controlled environment. The order of addition and different durations of stimuli may result in marked differences in, and ongoing competition between, the predominant gene expression signature such as antiinflammatory (green-filled square), proinflammatory (red-filled square), or mixed (red/green-filled square). The expected outcome gene signatures represent the measurement point, where cells are collected and mRNA levels of relevant GC target genes, with pro- and antiinflammatory roles, are determined. The priming effect becomes of particular relevance under circumstances of increased stress prior to the inflammatory insult. Only one example is given here, but many variations in timing are possible.

with GCs, TNF- $\alpha$ and activators of TLR2, -3 , and -4 drive SPHK1 expression (Figure 2) during lung inflammation. This cooperation is restricted to macrophages and is dependent on the p38 MAPKand mitogen- and stress-activated protein kinase 1 (MSK1) signaling pathway as well as on optimal GR dimerization (69). Furthermore, the expression of the serine protease inhibitor $\alpha 1$-antichymotrypsin (encoded by serpin A3), a secreted acute-phase protein that is active in many inflammatory diseases, was enhanced by simultaneous challenge with DEX and TNF- $\alpha$ (77). Finally, the pro-inflammatory cytokine IL-1 $\beta$ cooperates with DEX to induce the feedback regulator MKP1 (50-52), which inactivates pro-inflammatory MAPK signaling, and the feedforward anti-inflammatory regulator ZFP36 $(61,78,79)$. ZFP36 is itself upregulated by inflammatory stimuli and subsequently targets the AU-rich 3'-untranslated region of the TNF transcript to promote its degradation.

It seems counterintuitive for a transcriptional boost of particular anti-inflammatory proteins such as ZFP36 (78), MKP1 (79, 80), and SPHK1 (69) to depend on a pro-inflammatory MAPK pathway. Even more puzzling, MKP1 exerts its anti-inflammatory action through inhibition of MAPK signaling and thus halts the above-mentioned cooperative effects $(51,81)$. However, antiinflammatory actions of MAPK pathway components such as the p38 MAPK $(82,83)$ and the downstream MSK $(80)$ have previously been described in some forms of inflammation. Newton and colleagues recently reported that a DUSP1-dependent negative feedback control reduces the feedforward control mediated by ZFP36 (79). Although these interactions constitute an incoherent feedforward loop, wherein an activator regulates both a gene and a repressor of a gene, this loop appears to serve as a sensor to prevent exces- sive inflammation $(79,84)$. Incoherent loops are actually believed to be necessary for negative and positive pulse generation, accelerated responses, and sensing of fold change $(85,86)$. The continuous competition between feedback and feedforward control should be interpreted not by following separate paths, but as an integrated network, wherein the temporal interplay between competing regulatory processes dictates the ultimate inflammatory status. Loss of one factor (e.g., MKP1) will lead to other systems taking over, (e.g., ZFP36), allowing for an efficient dampening of the inflammatory process and ensuring the return to homeostasis (79).

The mechanisms described above indicate that anti-inflammatory pathways are indirectly affected by GR target proteins, the expression of which is controlled by transactivation or other gene upregulation mechanisms. These anti-inflammatory pathways are required to fully control inflammation.

\section{Acute versus chronic inflammation}

Conditions characterized by sustained inflammation, such as autoimmune disorders, typically require a chronic GC treatment regimen. It is unknown whether these diseases can be managed without eliciting GRE-dependent pathways that are detrimental, such as those contributing to increased blood glucose levels. Additionally, some acute inflammatory diseases such as sepsis remain refractory to GC treatment for reasons still not fully understood. In mouse models in which GR dimerization is compromised $\left(\mathrm{GR}^{\mathrm{dim} / \mathrm{dim}}\right)$, coping with inflammation becomes a life-threatening challenge (87). These findings support a framework wherein enhancement of GR dimerization may be suitable for the treatment of acute inflammatory disorders, while the induction of GR 
monomers (which prevents clinically problematic metabolic side effects) may be suitable for the management of chronic inflammatory diseases (Figure 1 and ref. 13). The reasoning for why GR monomers may be the way forward for chronic diseases includes not only the concept that the GR monomers can mediate classic transrepression (on NF- $\mathrm{BB}$ and $\mathrm{AP}-1$ ) but also the concept that GR monomers can drive mechanisms whereby GR can work via halfsite association to activate a subset of anti-inflammatory target genes. In contrast, palindromic GRE-instructed GR dimer binding remains a mechanism to avoid, especially when considering hyperglycemia as a GC-associated side effect that is known to rely on this particular GR dimer-dependent mechanism.

\section{Nongenomic mechanisms of GC signaling}

In addition to gene-targeted pathways, GCs exert rapid, nongenomic actions that do not require protein synthesis. For example, ligand binding to GR $\alpha$ not only induces activation of the receptor but also releases components of the multiprotein chaperone complex. These accessory proteins set in motion secondary signaling cascades such as inhibition of EGFR signaling through the actions of c-Src $(1,2,88)$. Several other nongenomic GC signaling mechanisms have been described, including signaling through a membrane-bound form of GR in human peripheral blood mononuclear cells (PBMCs) (89), interactions with or regulation of the subcellular localization of particular kinases $(90,91)$, regulation of apoptosis by mitochondrial translocation of the activated GR $\alpha$ in thymocytes (92), or translocation of GR $\alpha$ to caveolin-1-dependent lipid rafts, which affects cell proliferation $(93,94)$. Follow-up studies are warranted to further unravel the implications of these nongenomic GC-mediated actions.

\section{Potential pro-inflammatory actions of GCs}

In spite of the well-known net anti-inflammatory outcome of GR $\alpha$ actions, it is becoming increasingly clear that the receptor plays a dual role in immune gene regulation (Figure $3 \mathrm{~A}$ ). This notion is evidenced by GC-mediated enhancement of pro-inflammatory genes $(23,95)$, some examples of which are provided below.

Danger-associated molecular patterns and pattern recognition receptors. Endogenous molecules released following cellular damage or stress, e.g., extracellular ATP or uric acid crystals, are danger-associated molecular patterns (DAMPs) that can initiate innate immune responses by binding pattern recognition receptors (PRRs). The NLR family pyrin domain containing 3 (NLRP3) gene encodes an intracellular PRR (called NALP3) that is part of the NALP3 inflammasome, a multiprotein oligomer that also contains caspase protease family members. NALP3 detects products of damaged cells, after which the activated receptor triggers immune responses. In differentiated macrophages, GCs rapidly and directly enhance expression of NALP3. Exposure of GC-sensitized macrophages to extracellular ATP in combination with LPS, which is an inflammasome-activating signal, enhances the release of IL-1 $\beta$ and other cytokines. This finding highlights a novel role for GCs as sensitizers or priming agents of an initial inflammatory response in the innate immune system (96). Priming or sensitization commonly refers to sequential signals whereby exposure to the first signal exacerbates the response to the second signal. These signals may be of the same nature - for example, initial
cytokine/TLR signals triggering inflammatory responses may exacerbate inflammatory responses to cytokine/TLR signals in a second wave - or of different natures. In the field of neuroinflammation, a GC-mediated priming of innate immune cells was identified in the hippocampal microglia and possibly other CNS macrophages. Acute $(97,98)$ or chronic (99-101) exposure to exogenous or endogenous GCs increases NLRP3 mRNA expression in microglia. NLRP3 expression shifts the microglia activation via upregulation of myeloid markers, including MHC-II. Subsequent exposure to LPS potentiates microglial pro-inflammatory responses such as the secretion of TNF- $\alpha$, IL- $1 \beta$, and IL- 6 . As the endogenous or exogenous GCs alone fail to induce IL-1 $\beta$ expression, this augmentation reflects a priming of neuro-inflammatory processes wherein GCs act as an endogenous danger signal to prepare the organism to cope with subsequent immunological threats.

Specific intracellular proteins also serve as DAMPs. A possible mechanism for neuro-inflammatory priming may be the GCinduced release of DAMP molecules such as high mobility group box- 1 by damaged neurons, which is also actively secreted by innate immune cells. These findings on neuro-inflammation, together with the work on peripheral macrophages, indicate that macrophages throughout the body may be primed by GCs, regardless of their microenvironment $(96,101)$. Similarly, GCs mediate sensitization in human microvascular endothelial cells by transiently enhancing the expression of the purinergic GPCR P2Y $\mathrm{Y}_{2} \mathrm{R}$, which is activated by extracellular ATP. Exposure to extracellular ATP induces GC-primed cells to produce enhanced levels of inflammatory mediators such as IL-6, IL-8, and intercellular adhesion molecule-1. Remarkably, this increase is highly stimulus specific, as LPSinduced release of IL- 6 remains suppressed by GCs (73).

GCs also affect the activity of PRRs that react with pathogen-associated molecular patterns. For example, GCs cooperatively enhance TNF- $\alpha$-induced expression of TLR2 in the lung epithelial cell line A549. The mechanism for the GC/TNF- $\alpha$ cooperation includes an interaction between $\mathrm{GR} \alpha, \mathrm{NF}-\kappa \mathrm{B}$, and STAT 5 at the TLR2 promoter. This pro-inflammatory augmentation is gene specific, as NF- $\kappa \mathrm{B}$-driven proinflammatory $I l 8 \mathrm{mRNA}$ expression in the same cells under identical conditions is repressed by DEX. The TLR2 promoter region contains several GRE half-sites, which upon mutagenesis lead to the loss of cooperativity between GCs and TNF- $\alpha$ in transient TLR2 reporter assays (102). GCs in combination with a TLR2 agonist alter early signaling events of the TLR2 receptor. TLR2 phosphorylation is increased and Akt phosphorylation is diminished, via a mechanism involving a direct interaction between GR $\alpha$ and PI3K (103). GCs still induce anti-inflammatory pathways in parallel, such as the induction of MKP-1 in the A549 cells and TNFAIP3 (A20) in microvascular endothelial cells (51, $55,64,73,102)$. In DCs, GCs enhance the expression of both TLR2 and TLR4 but impair TLR-induced maturation and production of pro-inflammatory cytokines (21), indicating that TLR signaling is blocked further downstream of the receptor. Additionally, these findings demonstrate the cell specificity of GC-induced signaling: in endothelial cells GCs augment the TLR pathway, while in DCs GCs suppress effects downstream of the TLR itself. The interference of GR $\alpha$ with TLRs is a well-known anti-inflammatory action of GR, and GC-mediated regulation of endogenous inhibitors of TLR pathways could occur through several mechanisms, as 
described above and as summarized by Chinenov and Rogatsky (24). These mechanisms include the already described GC-mediated upregulation of MKP1, the upregulation of the suppressor of cytokine signaling 1 (SOCS1), which is able to inhibit JAK/STAT pathways triggered by both pro- and anti-inflammatory cytokines, and the inhibition of AP-1/NF- $\mathrm{BB}$ by the GILZ protein (see "Anti-inflammatory effects via gene activation" above).

Cytokine-induced gene expression by GCs. GCs coregulate genome-wide gene expression in concert with inflammatory mediators such as TNF- $\alpha$ (77), leukemia-initiating factor (LIF) (104), LPS (86), and IL-6 (105). The activity exerted by combined GCs and IL-6 can be pro-inflammatory, by mediating induction of acute-phase inflammatory proteins, as well as anti-inflammatory, by dampening IL-6-induced SOCS3 expression in primary hepatocytes (105). In the case of LIF, combined action with GCs enhances the induction of a class of genes involved in the hepatic acute-phase response and the innate cellular defense system. This particular augmentation of gene expression is mainly dependent on de novo protein synthesis and is thus categorized as a secondary response, delayed response, as relevant mediators need first to be synthesized before the innate cell defense response genes can be augmented (104). In macrophages, DEX and LPS coregulate several early genes, both pro- and anti-inflammatory, in a cooperative or antagonistic fashion. A number of these early genes code for transcriptional regulators such as members of the KLF family (86). Thus, GR $\alpha$, in combination with other TFs, triggers numerous feedforward loops and other regulatory networks, further increasing the complexity of gene regulation and leading to an anti- or pro-inflammatory outcome that is dependent on the timing of these gene responses.

Generally, when GCs act in a pro-inflammatory manner, they do so by activating components of the innate immune pathways, thereby priming the immune system under basal conditions to better control subsequent dangers. GCs further reinforce the innate immune system and acute-phase response during the early stages of inflammation by synergistically enhancing cytokinemediated gene expression. During ongoing inflammation, GCs exert systemic anti-inflammatory effects on the main pro-inflammatory pathways by repressing $\mathrm{NF}-\kappa \mathrm{B}$ signaling and inducing anti-inflammatory signaling proteins (e.g., MKP-1). Furthermore, GC actions on the adaptive immune system generally tend to be anti-inflammatory, preventing excessive inflammation and tissue damage and ultimately restoring homeostasis (84). This distinction between innate and adaptive immune effectors was also seen by Galon and colleagues in human PBMCs, wherein GCs induced the expression of innate immune-related genes, including scavenger and toll-like receptors, but repressed the expression of adaptive immune-related genes (95).

Relevant to the translation of the above findings to a more clinical setting is the finding that the upregulation of apparently pro-inflammatory cytokines, chemokines, and receptors is now firmly documented in the human airways in vivo following the inhalation of the GC budesonide (106). Taken together, opposite processes controlled both by GC-activated GR are installed to prepare the immune system to respond to a stressor (i.e., GC proinflammatory effects) and subsequently restore homeostasis (i.e., GC anti-inflammatory effects).
Priming and timing of GC responses. The sequence in which GC treatment and immune challenge follow each other further impacts the final inflammatory responses (Figure 3B). If GCs are added prior to the immune challenge, which is common practice in a laboratory environment, they potentiate inflammatory responses; but when added after the challenge, they suppress the pro-inflammatory response. This occurs both peripherally (liver) and centrally (hippocampus) (97). Several other studies are in line with this timing model $(73,100,105)$, including within the microvascular endothelium. Synergistic upregulation of pro-inflammatory genes is also observed when GCs and the immune challenge are administrated simultaneously $(22,77,86,102-104)$. GC exposure prior to inflammation can in some cases induce anti-inflammatory actions, as previously described in the suppression of bacteria-induced innate immune responses (65) and inhibition of microglial activation by DEX-activated GR $\alpha$ or by astragaloside IV (107). Similarly, GC administration during ongoing inflammation can still induce pro-inflammatory gene and protein expression (96). In an attempt to restore homeostasis, an enhanced neuro-inflammatory response following a priming signal may not always be harmless and the benefit may be highly context dependent. Several studies showed neuron death and worsening of neurodegenerative disorders as a consequence of the pro-inflammatory effects after prolonged GC exposure (108-111). Further studies are required to fully understand the actions and consequences of the dual role of GR $\alpha$ and to calculate the risk associated with the presence of high levels of stressinduced endogenous GCs or with exogenous GC treatments.

\section{Flipping the coin: anti- or pro-inflammatory outcomes}

GCs set in motion opposing forces by simultaneously inducing both pro- and anti-inflammatory pathways, ultimately producing a pro- or anti-inflammatory response as part of a pro-resolving and homeostasis-reaching strategy. The effect of GR $\alpha$ activation is highly gene specific, cell specific, and stimulus specific. Predicting the physiologic outcome of GC treatment is further complicated by secondary effects, as the receptor acts as a hub that influences numerous highly branched regulatory networks, which are themselves cell specific. Even within the same cell type, the effect of GCs can vary depending on the activation state of the cell (95), cofactor recruitment patterns (39), or differentiation stage (96). Additionally, the effects of GC exposure change over time due to changes in the expression of interacting components and the existence of various feedback and feedforward loops, among other mechanisms. The ability of GCs to downregulate their own receptor, a process known as homologous downregulation, is a physiologic phenomenon involving a decrease in GR mRNA and protein levels (112). This phenomenon can have wider consequences. For example, long-term exposure to GCs leads to compensatory $\mathrm{GR} \alpha$ downregulation in the frontal cortex and hippocampus. This downregulation accelerates neuro-inflammation via NLRP1 inflammasome activation and subsequent neuronal degeneration (108). Furthermore, the timing of treatment administration is of importance, as exemplified by a recent study revealing a regulatory mechanism linking the circadian clock and GC hormones together in the control of pulmonary inflammation and bacterial infection responses (113). The severity of the GC-inducing stress- 
or also affects the final outcome $(84,86)$. Contributing factors are summarized in Figure 3.

\section{GC side effects and resistance}

As most of the cooperative and synergistic effects between GCs and pro-inflammatory stimuli discovered so far ultimately promote anti-inflammatory programs or induce life-saving mechanisms at early stages of inflammation, chronic GC usage still precipitates many adverse side effects, including GC resistance. The list of GC-associated adverse effects, including osteoporosis, diabetes, glaucoma, skin atrophy, and depression is long due to the pleiotropic functions of the $\mathrm{GR} \alpha$ in various biological processes (Figure 1 and refs. 1, 19,111). Disruption of the naturally occurring circadian and ultradian changes in circulating endogenous GC levels with exogenous GCs or chronic stress breaks the delicate control systems and provokes unwanted effects (113-115). This dysregulation is particularly clear during treatment of arthritis, due to the essential role of GR in bone homeostasis, as reviewed in depth by Hartmann and colleagues (115).

Chronic GC treatment or chronic stress elicits tissue-specific GC resistance, causing diminished therapeutic capacity while often retaining detrimental side effects. Many studies point to a GC-mediated GR $\alpha$ downregulation as a primary mechanism for acquired resistance $(70,108,116-118)$. For example, in periodontal tissue that is inflamed due to chronic stress, the loss of GR $\alpha$ expression enhances Akt phosphorylation, which promotes TLR4 transcription and LPS-induced NF- $\kappa \mathrm{B}$ activation, consequently accelerating the pathologic progression of periodontitis (117). Some inflammatory disorders such as chronic obstructive pulmonary disease and cystic fibrosis are intrinsically GC resistant. Various mechanisms for resistance, including cytokine-induced upregulation of the dominant-negative inhibitor GR $\beta$ isoform, are summarized by Barnes $(119,120)$.

Continuous efforts to circumvent these two main problems of GC treatment are based on diverse strategies, including the use of selective GR ligands (121-124), targeting of the GR interactome (125), tissue-specific targeting, and strategies to influence posttranslational modifications and isoform expression (1). Several combination treatments have also been shown to be beneficial (49, 126-128), for example ginsenoside Rh1, which potentiates the long-term DEX-mediated anti-inflammatory effects in a collageninduced arthritis model while diminishing hyperglycemia (127).

\section{Future prospects}

Given the clinical need, novel GCs are still being brought to the market. These drugs differ in half-life, potency, or (for topical applications) the amount of systemic action. To design more refined drugs that influence GR signaling in a desired manner, it remains crucial to combine all layers of information in order to understand the complex mechanisms that govern GR activity. Herein we reviewed the integration of various signals by GRs, including those from other receptors involved in immune regulation. Interestingly, as recently reviewed by Hapgood et al. (129), this integration can even occur in the absence of GCs, contributing to a shift in the sensitivity of target cells to subsequent GC exposure.

As suggested by the Yamamoto team, future approaches to developing GC-based treatment strategies will necessitate gathering and integrating data on priming, (de)sensitization, and (in) activation of GR, as well as synergies and cross-talk with other signaling pathways, for each individual, allowing for a personalized medicine approach in the nearby future (130). Such studies will be made possible by a combination of interdisciplinary approaches in biological, physical, engineering, and computer and health sciences (130). Bearing in mind the above-described complexities of GR-mediated responses, future clinicians will have to make pragmatic choices informed by the knowledge being generated today. Patient stratification will become essential to decide on the need for a GR dimer-favoring drug (for acute disease) and GR monomer-favoring drug (for chronic disease) (13). Besides the dose and frequency of treatment, additional parameters may influence the healing process and may depend on a more precise timing of treatment (morning versus evening), the nutritional state of the patient, and disease dynamics that may require a switch to another GR-modulating drug at some point during the disease course.

\section{Acknowledgments}

SJD holds a Ph.D. fellowship of the Research Foundation - Flanders (FWO-Vlaanderen; FWO12-ASP-093). KDB was supported by a BOF ZAP grant B/13741/01. The authors apologize to colleagues whose work could not be included because of space constraints.

Address correspondence to: Karolien De Bosscher, Department of Biochemistry, Ghent University, Albert Baertsoenkaai 3, B-9000 Ghent, Belgium. Phone: 32.92649363; E-mail: karolien. debosscher@vib-ugent.be.
1. Ramamoorthy S, Cidlowski JA. Corticosteroids: mechanisms of action in health and disease. Rheum Dis Clin North Am. 2016;42(1):15-31.

2. Cain DW, Cidlowski JA. Specificity and sensitivity of glucocorticoid signaling in health and disease. Best Pract Res Clin Endocrinol Metab. 2015;29(4):545-556.

3. Desmet SJ, et al. The increasing complexity of glucocorticoid receptor signaling and regulation. Proc Belg Royal Acad Med. 2014;3:33-52.

4. Lu NZ, Cidlowski JA. Translational regulatory mechanisms generate $\mathrm{N}$-terminal glucocorticoid receptor isoforms with unique transcriptional target genes. Mol Cell. 2005;18(3):331-342.

5. Lu NZ, Collins JB, Grissom SF, Cidlowski JA. Selective regulation of bone cell apoptosis by translational isoforms of the glucocorticoid receptor. Mol Cell Biol. 2007;27(20):7143-7160.

6. Oakley RH, Cidlowski JA. Cellular processing of the glucocorticoid receptor gene and protein: new mechanisms for generating tissue-specific actions of glucocorticoids. J Biol Chem. 2011;286(5):3177-3184.

7. Grad I, Picard D. The glucocorticoid responses are shaped by molecular chaperones. Mol Cell Endocrinol. 2007;275(1-2):2-12.

8. Ebong IO, Beilsten-Edmands V, Patel NA, Morgner N, Robinson CV. The interchange of immunophilins leads to parallel pathways and different intermediates in the assembly of Hsp90 glucocorticoid receptor complexes. Cell Discov. 2016;2:16002.
9. Binder EB. The role of FKBP5, a co-chaperone of the glucocorticoid receptor in the pathogenesis and therapy of affective and anxiety disorders. Psychoneuroendocrinology. 2009;34(suppl 1):S186-S195.

10. Antunica-Noguerol M, et al. The activity of the glucocorticoid receptor is regulated by SUMO conjugation to FKBP51. Cell Death Differ. 2016;23(10):1579-1591.

11. So AY, Chaivorapol C, Bolton EC, Li H, Yamamoto KR. Determinants of cell- and gene-specific transcriptional regulation by the glucocorticoid receptor. PLoS Genet. 2007;3(6):e94.

12. De Bosscher K, Vanden Berghe W, Haegeman G. The interplay between the glucocorticoid reeceptor and nuclear factor- $\kappa \mathrm{B}$ or activator protein-1: molecular mechanisms for gene repression. 
Endocr Rev. 2003;24(4):488-522.

13. De Bosscher K, Beck IM, Ratman D, Berghe WV, Libert C. Activation of the glucocorticoid receptor in acute inflammation: the SEDIGRAM Concept. Trends Pharmacol Sci. 2016;37(1):4-16.

14. Fölster-Holst R, Abeck D, Torrelo A. Topical hydrocortisone 17-butyrate 21-propionate in the treatment of inflammatory skin diseases: pharmacological data, clinical efficacy, safety and calculation of the therapeutic index. Pharmazie. 2016;71(3):115-121.

15. Badorrek P, Hohlfeld JM, Krug N, Joshi A, Raut A. Efficacy and safety of a novel nasal steroid, S0597, in patients with seasonal allergic rhinitis. Ann Allergy Asthma Immunol. 2015;115(4):325-329.e1.

16. Le Page E, et al. Oral versus intravenous highdose methylprednisolone for treatment of relapses in patients with multiple sclerosis (COPOUSEP): a randomised, controlled, double-blind, non-inferiority trial. Lancet. 2015;386(9997):974-981.

17. Barnes PJ. Severe asthma: advances in current management and future therapy. JAllergy Clin Immunol. 2012;129(1):48-59.

18. Strehl C, et al. Defining conditions where longterm glucocorticoid treatment has an acceptably low level of harm to facilitate implementation of existing recommendations: viewpoints from an EULAR task force. Ann Rheum Dis. 2016;75(6):952-957.

19. Schäcke H, Döcke WD, Asadullah K. Mechanisms involved in the side effects of glucocorticoids. Pharmacol Ther. 2002;96(1):23-43.

20. Theiss-Suennemann J, et al. Glucocorticoids attenuate acute graft-versus-host disease by suppressing the cytotoxic capacity of CD8(+) T cells. J Pathol. 2015;235(4):646-655.

21. Rozkova D, Horvath R, Bartunkova J, Spisek R. Glucocorticoids severely impair differentiation and antigen presenting function of dendritic cells despite upregulation of Toll-like receptors. Clin Immunol. 2006;120(3):260-271.

22. Dejager L, Vandevyver S, Petta I, Libert C. Dominance of the strongest: inflammatory cytokines versus glucocorticoids. Cytokine Growth Factor Rev. 2014;25(1):21-33.

23. Cruz-Topete D, Cidlowski JA. One hormone, two actions: anti- and pro-inflammatory effects of glucocorticoids. Neuroimmunomodulation. 2015;22(1-2):20-32.

24. Chinenov Y, Rogatsky I. Glucocorticoids and the innate immune system: crosstalk with the tolllike receptor signaling network. Mol Cell Endocrinol. 2007;275(1-2):30-42.

25. Chinenov Y, Gupte R, Rogatsky I. Nuclear receptors in inflammation control: repression by GR and beyond. Mol Cell Endocrinol. 2013;380(1-2):55-64.

26. Liden J, Delaunay F, Rafter I, Gustafsson J, Okret S. A new function for the C-terminal zinc finger of the glucocorticoid receptor. Repression of RelA transactivation. JBiol Chem. 1997;272(34):21467-21472.

27. Louw-du Toit R, Hapgood JP, Africander D. Medroxyprogesterone acetate differentially regulates interleukin (IL)-12 and IL-10 in a human ectocervical epithelial cell line in a glucocorticoid receptor (GR)-dependent manner. J Biol Chem. 2014;289(45):31136-31149.
28. Scheinman RI, Gualberto A, Jewell CM, Cidlowski JA, Baldwin AS. Characterization of mechanisms involved in transrepression of NF- $\kappa \mathrm{B}$ by activated glucocorticoid receptors. Mol Cell Biol. 1995;15(2):943-953.

29. Ito K, et al. Histone deacetylase 2-mediated deacetylation of the glucocorticoid receptor enables NF- $\kappa \mathrm{B}$ suppression. J Exp Med. 2006;203(1):7-13.

30. Ogawa S, et al. Molecular determinants of crosstalk between nuclear receptors and toll-like receptors. Cell. 2005;122(5):707-721.

31. Nissen RM, Yamamoto KR. The glucocorticoid receptor inhibits NF- $\kappa \mathrm{B}$ by interfering with serine-2 phosphorylation of the RNA polymerase II carboxy-terminal domain. Genes Dev. 2000;14(18):2314-2329.

32. Luecke HF, Yamamoto KR. The glucocorticoid receptor blocks P-TEFb recruitment by NF- $\kappa \mathrm{B}$ to effect promoter-specific transcriptional repression. Genes Dev. 2005;19(9):1116-1127.

33. Gupte R, Muse GW, Chinenov Y, Adelman K, Rogatsky I. Glucocorticoid receptor represses proinflammatory genes at distinct steps of the transcription cycle. Proc Natl Acad Sci U S A. 2013;110(36):14616-14621.

34. Coppo M, Chinenov Y, Sacta MA, Rogatsky I. The transcriptional coregulator GRIP1 controls macrophage polarization and metabolic homeostasis. Nat Commun. 2016;7:12254

35. McKay LI, Cidlowski JA. CBP (CREB binding protein) integrates NF-kappaB (nuclear factor- $\mathrm{kB}$ ) and glucocorticoid receptor physical interactions and antagonism. Mol Endocrinol. 2000;14(8):1222-1234.

36. Sheppard KA, et al. Nuclear integration of glucocorticoid receptor and nuclear factor $-\kappa \mathrm{B}$ signaling by CREB-binding protein and steroid receptor coactivator-1.J Biol Chem. 1998;273(45):29291-29294.

37. De Bosscher K, Vanden Berghe W, Vermeulen L, Plaisance S, Boone E, Haegeman G. Glucocorticoids repress NF- $\mathrm{kB}$-driven genes by disturbing the interaction of $\mathrm{p} 65$ with the basal transcription machinery, irrespective of coactivator levels in the cell. Proc Natl Acad Sci US A. 2000;97(8):3919-3924.

38. De Bosscher K, Vanden Berghe W, Haegeman G. Glucocorticoid repression of AP-1 is not mediated by competition for nuclear coactivators. $\mathrm{Mol}$ Endocrinol. 2001;15(2):219-227.

39. Chinenov Y, et al. Role of transcriptional coregulator GRIP1 in the anti-inflammatory actions of glucocorticoids. Proc Natl Acad Sci U S A. 2012;109(29):11776-11781.

40. Reily MM, Pantoja C, Hu X, Chinenov Y, Rogatsky I. The GRIP1:IRF3 interaction as a target for glucocorticoid receptor-mediated immunosuppression. EMBO J. 2006;25(1):108-117.

41. Murphy SH, et al. Tumor suppressor protein (p)53, is a regulator of NF-kappaB repression by the glucocorticoid receptor. Proc Natl Acad Sci US A. 2011;108(41):17117-17122.

42. Schiller BJ, Chodankar R, Watson LC, Stallcup MR, Yamamoto KR. Glucocorticoid receptor binds half sites as a monomer and regulates specific target genes. Genome Biol. 2014;15(7):418.

43. Lim HW, et al. Genomic redistribution of GR monomers and dimers mediates transcriptional response to exogenous glucocorticoid in vivo. Genome Res. 2015;25(6):836-844.

44. Biddie SC, et al. Transcription factor AP1 potentiates chromatin accessibility and glucocorticoid receptor binding. Mol Cell. 2011;43(1):145-155.

45. Barnes PJ. Anti-inflammatory actions of glucocorticoids: molecular mechanisms. Clin Sci. 1998;94(6):557-572.

46. King EM, Chivers JE, Rider CF, Minnich A, Giembycz MA, Newton R. Glucocorticoid repression of inflammatory gene expression shows differential responsiveness by transactivation- and transrepression-dependent mechanisms. PLoS One. 2013;8(1):e53936.

47. Clark AR, Belvisi MG. Maps and legends: the quest for dissociated ligands of the glucocorticoid receptor. Pharmacol Ther. 2012;134(1):54-67.

48. Vandevyver S, Dejager L, Tuckermann J, Libert C. New insights into the anti-inflammatory mechanisms of glucocorticoids: an emerging role for glucocorticoid-receptor-mediated transactivation. Endocrinology. 2013;154(3):993-1007.

49. Newton R. Anti-inflammatory glucocorticoids: changing concepts. Eur J Pharmacol. 2014;724:231-236.

50. Lasa M, Abraham SM, Boucheron C, Saklatvala J, Clark AR. Dexamethasone causes sustained expression of mitogen-activated protein kinase (MAPK) phosphatase 1 and phosphatasemediated inhibition of MAPK p38. Mol Cell Biol. 2002;22(22):7802-7811.

51. Shah S, King EM, Chandrasekhar A, Newton R. Roles for the mitogen-activated protein kinase (MAPK) phosphatase, DUSP1, in feedback control of inflammatory gene expression and repression by dexamethasone. J Biol Chem. 2014;289(19):13667-13679.

52. Kassel O, Sancono A, Krätzschmar J, Kreft B, Stassen M, Cato AC. Glucocorticoids inhibit MAP kinase via increased expression and decreased degradation of MKP-1. EMBO J. 2001;20(24):7108-7116.

53. Fürst R, et al. MAPK phosphatase-1 represents a novel anti-inflammatory target of glucocorticoids in the human endothelium. FASEB J. 2007;21(1):74-80.

54. Abraham SM, et al. Antiinflammatory effects of dexamethasone are partly dependent on induction of dual specificity phosphatase 1. J Exp Med. 2006;203(8):1883-1889.

55. Joanny E, Ding Q, Gong L, Kong P, Saklatvala J, Clark AR. Anti-inflammatory effects of selective glucocorticoid receptor modulators are partially dependent on up-regulation of dual specificity phosphatase 1 . Br J Pharmacol. 2012;165(4b):1124-1136.

56. Tchen CR, Martins JR, Paktiawal N, Perelli R, Saklatvala J, Clark AR. Glucocorticoid regulation of mouse and human dual specificity phosphatase 1 (DUSP1) genes: unusual cis-acting elements and unexpected evolutionary divergence. J Biol Chem. 2010;285(4):2642-2652.

57. Reichardt HM, et al. DNA binding of the glucocorticoid receptor is not essential for survival. Cell.1998;93(4):531-541.

58. Vandevyver S, et al. Glucocorticoid receptor dimerization induces MKP1 to protect against 
TNF-induced inflammation. J Clin Invest. 2012;122(6):2130-2140.

59. Auphan N, DiDonato JA, Rosette C, Helmberg A, Karin M. Immunosuppression by glucocorticoids: inhibition of NF- $\mathrm{BB}$ activity through induction of IkB sythesis. Science. 1995;270(5234):286-290.

60. Scheinman RI, Cogswell PC, Lofquist AK, Baldwin AS. Role of transcriptional activation of IkBa in mediation of immunosupression by glucocorticoids. Science. 1995;270(5234):283-286.

61. Smoak K, Cidlowski JA. Glucocorticoids regulate tristetraprolin synthesis and posttranscriptionally regulate tumor necrosis factor alpha inflammatory signaling. Mol Cell Biol. 2006;26(23):9126-9135.

62. De Bosscher K, Schmitz ML, Vanden Berghe W, Plaisance S, Fiers W, Haegeman G. Glucocorticoid-mediated repression of nuclear factor- $\mathrm{\kappa B}-$ dependent transcription involves direct interference with transactivation. Proc Natl Acad Sci US A. 1997;94(25):13504-13509.

63. Morjaria JB, Babu KS, Polosa R, Holgate ST. Tumor necrosis factor- $\alpha$ in severe corticosteroid-refractory asthma. Expert Rev Respir Med. 2007;1(1):51-63.

64. Altonsy MO, Sasse SK, Phang TL, Gerber AN. Context-dependent cooperation between nuclear factor $\kappa \mathrm{B}(\mathrm{NF}-\kappa \mathrm{B})$ and the glucocorticoid receptor at a TNFAIP3 intronic enhancer: a mechanism to maintain negative feedback control of inflammation. J Biol Chem. 2014;289(12):8231-8239.

65. Miyata M, et al. Glucocorticoids suppress inflammation via the upregulation of negative regulator IRAK-M. Nat Commun. 2015;6:6062.

66. Kobayashi K, Hernandez LD, Galán JE, Janeway CA, Medzhitov R, Flavell RA. IRAK-M is a negative regulator of Toll-like receptor signaling. Cell. 2002;110(2):191-202.

67. De Bosscher K, et al. A fully dissociated compound of plant origin for inflammatory gene repression. Proc Natl Acad Sci US A. 2005;102(44):15827-15832.

68. Dewint $P$, et al. A plant-derived ligand favoring monomeric glucocorticoid receptor conformation with impaired transactivation potential attenuates collagen-induced arthritis. J Immunol. 2008;180(4):2608-2615.

69. Vettorazzi S, et al. Glucocorticoids limit acute lung inflammation in concert with inflammatory stimuli by induction of SphK1. Nat Commun. 2015;6:7796.

70. Robert O, et al. Decreased expression of the glucocorticoid receptor-GILZ pathway in Kupffer cells promotes liver inflammation in obese mice. J Hepatol. 2016;64(4):916-924.

71. Ronchetti S, Migliorati G, Riccardi C. GILZ as a mediator of the anti-inflammatory effects of glucocorticoids. Front Endocrinol (Lausanne). 2015;6:170.

72. Rebbe NF, Hickman S. Modulation of nucleotide pyrophosphatase in plasmacytoma cells. Biochem Biophys Res Commun. 1991;175(2):637-644.

73. Ding Y, Gao ZG, Jacobson KA, Suffredini AF. Dexamethasone enhances ATP-induced inflammatory responses in endothelial cells. J Pharmacol Exp Ther. 2010;335(3):693-702.

74. Patel HB, et al. The impact of endogenous annexin A1 on glucocorticoid control of inflammatory arthritis. Ann Rheum Dis. 2012;71(11):1872-1880.

75. Abbinante-Nissen JM, Simpson LG, Leikauf GD.

Corticosteroids increase secretory leukocyte protease inhibitor transcript levels in airway epithelial cells. Am J Physiol. 1995;268(4 pt 1):L601-L606.

76. Kadiyala V, et al. Cistrome-based cooperation between airway epithelial glucocorticoid receptor and NF- $\kappa$ B orchestrates anti-inflammatory effects. J Biol Chem. 2016;291(24):12673-12687.

77. Lannan EA, Galliher-Beckley AJ, Scoltock AB, Cidlowski JA. Proinflammatory actions of glucocorticoids: glucocorticoids and TNF $\alpha$ coregulate gene expression in vitro and in vivo. Endocrinology. 2012;153(8):3701-3712.

78. King EM, Kaur M, Gong W, Rider CF, Holden NS, Newton R. Regulation of tristetraprolin expression by interleukin- $1 \beta$ and dexamethasone in human pulmonary epithelial cells: roles for nuclear factor- $\kappa \mathrm{B}$ and p38 mitogenactivated protein kinase. JPharmacol Exp Ther. 2009;330(2):575-585.

79. Shah S, Mostafa MM, McWhae A, Traves SL, Newton R. Negative feed-forward control of tumor necrosis factor (TNF) by tristetraprolin (ZFP36) is limited by the mitogenactivated protein kinase phosphatase, dual-specificity phosphatase 1 (DUSP1): implications for regulation by glucocorticoids. J Biol Chem. 2016;291(1):110-125.

80. Ananieva O, et al. The kinases MSK1 and MSK2 act as negative regulators of Toll-like receptor signaling. Nat Immunol. 2008;9(9):1028-1036.

81. Huotari N, Hömmö T, Taimi V, Nieminen R, Moilanen E, Korhonen R. Regulation of tristetraprolin expression by mitogen-activated protein kinase phosphatase-1. APMIS. 2012;120(12):988-999.

82. Guma M, et al. Antiinflammatory functions of p38 in mouse models of rheumatoid arthritis: Advantages of targeting upstream kinases MKK-3 or MKK-6. Arthritis Rheumatol. 2012;64(9):2887-2895.

83. Kim C, et al. The kinase p $38 \alpha$ serves cell type-specific inflammatory functions in skin injury and coordinates pro- and anti-inflammatory gene expression. Nat Immunol. 2008;9(9):1019-1027.

84. Busillo JM, Cidlowski JA. The five Rs of glucocorticoid action during inflammation: ready, reinforce, repress, resolve, and restore. Trends Endocrinol Metab. 2013;24(3):109-119.

85. Goentoro L, Shoval O, Kirschner MW, Alon U. The incoherent feedforward loop can provide fold-change detection in gene regulation. $\mathrm{Mol}$ Cell. 2009;36(5):894-899.

86. Chinenov Y, Coppo M, Gupte R, Sacta MA, Rogatsky I. Glucocorticoid receptor coordinates transcription factor-dominated regulatory network in macrophages. BMC Genomics. 2014;15:656.

87. Kleiman A, et al. Glucocorticoid receptor dimerization is required for survival in septic shock via suppression of interleukin-1 in macrophages. FASEB J. 2012;26(2):722-729.

88. Croxtall JD, Choudhury Q, Flower RJ. Glucocorticoids act within minutes to inhibit recruitment of signalling factors to activated EGF receptors through a receptor-dependent, transcription-independent mechanism. Br J Pharmacol. 2000;130(2):289-298.

89. Bartholome B, et al. Membrane glucocorticoid receptors (mGCR) are expressed in normal human peripheral blood mononuclear cells and up-regulated after in vitro stimulation and in patients with rheumatoid arthritis. FASEB J. 2004;18(1):70-80.

90. Ayroldi E, Cannarile L, Migliorati G, Nocentini G, Delfino DV, Riccardi C. Mechanisms of the anti-inflammatory effects of glucocorticoids: genomic and nongenomic interference with MAPK signaling pathways. FASEB J. 2012;26(12):4805-4820.

91. Beck IM, et al. Altered subcellular distribution of MSK1 induced by glucocorticoids contributes to NF- $\mathrm{KB}$ inhibition. EMBO J. 2008;27(12):1682-1693.

92. Talabér G, et al. Mitochondrial translocation of the glucocorticoid receptor in double-positive thymocytes correlates with their sensitivity to glucocorticoid-induced apoptosis. Int Immunol. 2009;21(11):1269-1276.

93. Matthews L, Berry A, Ohanian V, Ohanian J, Garside H, Ray D. Caveolin mediates rapid glucocorticoid effects and couples glucocorticoid action to the antiproliferative program. Mol Endocrinol. 2008;22(6):1320-1330.

94. Samarasinghe RA, et al. Nongenomic glucocorticoid receptor action regulates gap junction intercellular communication and neural progenitor cell proliferation. Proc Natl Acad Sci U S A. 2011;108(40):16657-16662.

95. Galon J, et al. Gene profiling reveals unknown enhancing and suppressive actions of glucocorticoids on immune cells. FASEB J. 2002;16(1):61-71.

96. Busillo JM, Azzam KM, Cidlowski JA. Glucocorticoids sensitize the innate immune system through regulation of the NLRP3 inflammasome. J Biol Chem. 2011;286(44):38703-38713.

97. Frank MG, Miguel ZD, Watkins LR, Maier SF. Prior exposure to glucocorticoids sensitizes the neuroinflammatory and peripheral inflammatory responses to $E$. coli lipopolysaccharide. Brain Behav Immun. 2010;24(1):19-30.

98. Frank MG, Thompson BM, Watkins LR, Maier SF. Glucocorticoids mediate stress-induced priming of microglial pro-inflammatory responses. Brain Behav Immun. 2012;26(2):337-345.

99. Espinosa-Oliva AM, et al. Stress is critical for LPS-induced activation of microglia and damage in the rat hippocampus. Neurobiol Aging. 2011;32(1):85-102.

100.Munhoz CD, et al. Chronic unpredictable stress exacerbates lipopolysaccharide-induced activation of nuclear factor- $\kappa \mathrm{B}$ in the frontal cortex and hippocampus via glucocorticoid secretion. J Neurosci. 2006;26(14):3813-3820.

101.Frank MG, Hershman SA, Weber MD, Watkins LR, Maier SF. Chronic exposure to exogenous glucocorticoids primes microglia to pro-inflammatory stimuli and induces NLRP3 mRNA in the hippocampus. Psychoneuroendocrinology. 2014;40:191-200.

102. Hermoso MA, Matsuguchi T, Smoak K, Cidlowski JA. Glucocorticoids and tumor necrosis factor alpha cooperatively regulate toll-like receptor 2 gene expression. Mol Cell Biol. 2004;24(11):4743-4756.

103. Arancibia S, et al. Phosphatidylinositol 3-kinase interacts with the glucocorticoid 
receptor upon TLR2 activation. J Cell Mol Med. 2011;15(2):339-349.

104.Langlais D, Couture C, Balsalobre A, Drouin J. Regulatory network analyses reveal genomewide potentiation of LIF signaling by glucocorticoids and define an innate cell defense response. PLoS Genet. 2008;4(10):e1000224.

105. Dittrich A, et al. Glucocorticoids increase interleukin-6-dependent gene induction by interfering with the expression of the suppressor of cytokine signaling 3 feedback inhibitor. Hepatology. 2012;55(1):256-266

106.Leigh R, et al. An inhaled dose of budesonide induces genes involved in transcription and signaling in the human airways: enhancement of anti- and proinflammatory effector genes. Pharmacol Res Perspect. 2016;4(4):e00243.

107. Liu HS, et al. Astragaloside IV inhibits microglia activation via glucocorticoid receptor mediated signaling pathway. Sci Rep. 2016;6:19137.

108. Hu W, et al. Chronic glucocorticoids exposure enhances neurodegeneration in the frontal cortex and hippocampus via NLRP-1 inflammasome activation in male mice. Brain Behav Immun. 2016;52:58-70.

109. Sorrells SF, Munhoz CD, Manley NC, Yen S, Sapolsky RM. Glucocorticoids increase excitotoxic injury and inflammation in the hippocampus of adult male rats. Neuroendocrinology. 2014;100(2-3):129-140.

110.Sorrells SF, et al. Glucocorticoid signaling in myeloid cells worsens acute CNS injury and inflammation. J Neurosci. 2013;33(18):7877-7889.

111. Voorhees JL, et al. Prolonged restraint stress increases IL-6, reduces IL-10, and causes persistent depressive-like behavior that is reversed by recombinant IL-10. PLoS One. 2013;8(3):e58488.

112. Wallace AD, Cao Y, Chandramouleeswaran S, Cidlowski JA. Lysine 419 targets human gluco- corticoid receptor for proteasomal degradation. Steroids. 2010;75(12):1016-1023.

113. Gibbs J, et al. An epithelial circadian clock controls pulmonary inflammation and glucocorticoid action. Nat Med. 2014;20(8):919-926.

114. Stavreva DA, et al. Ultradian hormone stimulation induces glucocorticoid receptor-mediated pulses of gene transcription. Nat Cell Biol. 2009;11(9):1093-1102.

115. Hartmann K, et al. Molecular actions of glucocorticoids in cartilage and bone during health, disease, and steroid therapy. Physiol Rev. 2016;96(2):409-447.

116. Gossye V, Elewaut D, Van Beneden K, Dewint P, Haegeman G, De Bosscher K. A plant-derived glucocorticoid receptor modulator attenuates inflammation without provoking ligand-induced resistance. Ann Rheum Dis. 2010;69(1):291-296.

117. Lu H, et al. Chronic stress accelerates ligature-induced periodontitis by suppressing glucocorticoid receptor- $\alpha$ signaling. Exp Mol Med. 2016;48:e223.

118. Tessel MA, Benham AL, Krett NL, Rosen ST, Gunaratne PH. Role for microRNAs in regulating glucocorticoid response and resistance in multiple myeloma. Horm Cancer. 2011;2(3):182-189.

119. Barnes PJ. Corticosteroid resistance in patients with asthma and chronic obstructive pulmonary disease. JAllergy Clin Immunol. 2013;131(3):636-645.

120.Barnes PJ. Glucocorticosteroids: current and future directions. Br JPharmacol. 2011;163(1):29-43.

121. De Bosscher K, et al. Selective modulation of the glucocorticoid receptor can distinguish between transrepression of NF- $\mathrm{KB}$ and AP-1. Cell Mol Life Sci. 2014;71(1):143-163.

122. Rauch A, et al. An anti-inflammatory selective glucocorticoid receptor modulator preserves osteoblast differentiation. FASEB J.
2011;25(4):1323-1332.

123. Rauner M, et al. Dissociation of osteogenic and immunological effects by the selective glucocorticoid receptor agonist, compound $\mathrm{A}$, in human bone marrow stromal cells. Endocrinology. 2011;152(1):103-112.

124. Rauner M, et al. Effects of the selective glucocorticoid receptor modulator compound A on bone metabolism and inflammation in male mice with collagen-induced arthritis. Endocrinology. 2013;154(10):3719-3728.

125. Greulich F, Hemmer MC, Rollins DA, Rogatsky I, Uhlenhaut NH. There goes the neighborhood: Assembly of transcriptional complexes during the regulation of metabolism and inflammation by the glucocorticoid receptor. Steroids. 2016;114:7-15.

126. Bougarne N, et al. PPAR $\alpha$ blocks glucocorticoid receptor alpha-mediated transactivation but cooperates with the activated glucocorticoid receptor $\alpha$ for transrepression on NF-кB. Proc Natl Acad Sci U S A. 2009;106(18):7397-7402.

127. Li J, et al. Ginsenoside Rh1 potentiates dexamethasone's anti-inflammatory effects for chronic inflammatory disease by reversing dexamethasone-induced resistance. Arthritis Res Ther. 2014;16(3):R106.

128. Grundy S, Plumb J, Kaur M, Ray D, Singh D. Additive anti-inflammatory effects of corticosteroids and phosphodiesterase-4 inhibitors in COPD CD8 cells. Respir Res. 2016;17:9.

129. Hapgood JP, Avenant C, Moliki JM. Glucocorticoid-independent modulation of GR activity: Implications for immunotherapy. Pharmacol Ther. 2016;165:93-113.

130. Hawgood S, Hook-Barnard IG, O'Brien TC, Yamamoto KR. Precision medicine: Beyond the infection point. Sci Transl Med. 2015;7(300):300ps17. 